\title{
EVALUATION OF LEFT ATRIAL FUNCTION BY 2D SPECKLE TRACKING ECHOCARDIOGRAPHY IN PATIENTS WITH SYSTEMIC LUPUS ERYTHEMATOSUS
}

\author{
By \\ Abd El-Kawy Abd El-Halem Ahmed, Mamdouh Helmy El-Tahan, \\ Abdul-Hameed Abdul-Hareth Ghazaly* and Al-Sayed Ali Abdou Al- \\ Marghany
}

Departments of Cardiology and Physical medicine, Rheumatology and Rehabilitation*, Faculty of Medicine, Al-Azhar University

Corresponding author: Abd El-Kawy Abd El-Halem Ahmed,

E-mail: abdelkawy.abdelhalem@gmail.com

\begin{abstract}
Background: Left atrial (LA) speckle tracking echocardiography (STE) is a newer echocardiographic technique for strain and strain rate analyses that tracks 'speckles' or natural acoustic markers in the twodimensional (2D) ultrasound image. STE has been described for assessment of regional and global LA function. Systemic Lupus Erythematosus (SLE) is a chronic autoimmune disease that is characterized by inflammation and damage to multiple organ systems. Cardiac involvement can be seen in over $50 \%$ of lupus patients.
\end{abstract}

Objective: To evaluate LA function and volume by STE-derived strain and strain rate in SLE patients compared to controls and assess the relationship of LA function and varying grades of Left ventricular diastolic dysfunction (LVDD) in SLE patients.

Patients and Methods: Sixty SLE patients aged 20 to 40 with normal LV systolic function (LVEF > 50\%). SLE patients met the 2012 ACR revised criteria for SLE, were enrolled in this study. Age and sex matched apparently healthy forty subjects were included in this study as controls. Both of patients and control groups were selected from the attendants of Rheumatology Department and Cardiology Department of Al-Azhar University hospitals.

Results: No statistically significant difference between patients and control groups as regarding age, gender, body surface area (BSA), systolic blood pressure (SBP) and Diastolic blood pressure (DBP). The Left atrial volume index (LAVI) was significantly larger in the SLE patients group than that in control group. Diastolic function was normal in $31.66 \%$, impaired relaxation in $26.7 \%$, pseudo normal in $38.33 \%$ and restrictive in $3.34 \%$. Global strain, derived as an average of all segments, was $25.80 \pm 6.33$ in SLE group vs. $33.12 \pm 8.71$ in control group. Septal, lateral and apical longitudinal strains reduced in SLE patients with significantly differences between both groups.LA SRa increased in SLE group compared with that in control group $(1.97 \pm 0.47$ vs $1.57 \pm 0.60)$. There were significant differences in LAVI, SRs, SRe and SRa among normal and abnormal diastolic function in patients of the SLE group. The correlation between LVDD and left atrial SRs, SRe and, SRa showed that there were significant correlations with SRs, SRe but not significant with SRa.

Conclusion: LA function impaired in SLE patients. Left atrial remodeling and impaired LA mechanical functions can be detected accurately with speckle tracking echocardiography. Assessment of LA strain represents a simple, accurate and reproducible technique to evaluate LA function.

Keywords: Lupus erythematosus, Left atrial, LVDD, Echocardiography. 


\section{INTRODUCTION}

Cardiovascular disease (CVD) is being diagnosed with increasing frequency and is currently detected in up to 50\% of SLE patients. All cardiac structures may be involved, including the pericardium, endocardium, myocardium, coronary arteries and conductive tissue (Bartels et al., 2014). Compared to the general population, Systemic Lupus Erythematosus (SLE) patients have a 2 to 9-fold higher risk of cardiovascular events, such as myocardial infarction, stroke and heart failure (Hesselvig et al., 2017).

The long-term inflammatory burden and immune abnormality can result in serious damage to the heart. It has been reported that left ventricular diastolic dysfunction (LVDD) is common, early and persistent in SLE patients, and may be a manifestation of cardiac involvement preceding systolic dysfunction (Shang et al., 2012). LVDD elevates left intraventricular pressure during diastole, increasing the load on the left atrium (LA) and inducing the LA remodeling (Hsiao et al., 2012).

The LA contributes to cardiac hemodynamics by modulating LV filling through the interplay of atrial reservoir, conduit, and booster contractile function. The LA has additionally been identified as an important biomarker of cardiovascular disease and adverse cardiovascular outcomes. The noninvasive assessment of LA anatomy and function has been performed predominantly by echocardiographic volumetric and Doppler analyses (Vieira et al., 2014).

LA speckle tracking echocardiography (STE) is a newer echocardiographic technique for strain and strain rate analyses that tracks 'speckles' or natural acoustic markers in the two-dimensional (2D) ultrasound image. The geometric shift of each speckle position is traced throughout the cardiac cycle (Vieira et al., 2014). Recently, STE has been described for assessment of regional and global LA function (Vianna-Pinton et al., 2010). By the STE, early detection of subclinical LA dysfunction plays a crucial role in the evaluation of many cardiac diseases. Atrial strain has now been evaluated in multiple conditions, including hypertension, diabetes, heart failure, ischemic and valvular heart disease, and atrial fibrillation (Liu et al., 2014), including the facilitation of stroke risk calculation and assessment of prognostic implications (Obokata et al., 2014).

The aim of this study was to evaluate LA function and volume by STE-derived strain and strain rate in SLE patients compared to controls and assess the relationship of LA function and varying grades of LVDD in SLE patients.

\section{SUBJECTS AND METHODS}

Sixty SLE patients aged 20 to 40 with normal LV systolic function (LVEF > 50\%). SLE patients met the 2012 ACR revised criteria for SLE, were enrolled in this study. Age and sex matched apparently healthy forty subjects were included in this study as controls. Both of patients and control groups were selected from the attendants of Rheumatology and Cardiology Departments, Al-Azhar University Hospitals from October 2018 to January 2020. Informed consents were taken from patients and control after full explanation of the purpose and nature of the study. 


\section{Subjects were divided into two groups:}

1. Group I (Patient group) included (60) individuals with SLE. All patients fulfilled the American College of Rheumatology (ACR) diagnostic criteria of SLE (ACR, 2012).

2. Group II (Control subjects) included (40) healthy individuals.

\section{Exclusion criteria:}

- Significant valvular disease.

- Atrial fibrillation.

- Pericardial heart disease.

- Patients with prosthetic mitral valve.

- Patients with LV systolic dysfunction (EF $<50 \%)$.

- Patients with bad echo window or who completed study cannot be performed.

All the Patients and controls were subjected to the following:

\section{HISTORY TAKING:}

- Full medical history with special emphasis on:

1. Anthropometric data (age, gender, body weight).

2. History of diabetes mellitus (DM), hypertension and dyslipidemia, diabetes mellitus, hypertension, hypercholesterolemia, cigarette smoking.

3. Family history of premature coronary artery disease (CAD).

II. EXAMINATION:

General

examination and local examination.

\section{III.INVESTIGATIONS:}

The most recent laboratory testing was looked for in the medical files and recorded including:

1. Routine

Laboratory

Investigations: Complete blood count, erythrocyte sedimentation rate, C - reactive protein, renal function tests and complete urine analysis.

\section{Immunological investigations:}

- ANA test by immunofluorescence technique.

- Anti-ds DNA antibodies by immunofluorescence technique.

\section{Conventional Echocardiography:}

Conventional echocardiograms were acquired using a Philips ultrasound machine (Philips Medical Systems) with a broad band transducer (S5-1, 2-5MHz). All study participants were evaluated by two-dimensional echocardiography. LA dimension (LAD), left ventricular end diastolic diameter (LVEDD) and end systolic diameter (LVESD) were measured using $M$ mode from the parasternal long axis view. LA volume was measured from standard apical 4chamber views at end-systole just before mitral valve opening. LA borders were traced using planimetry in control and study subjects. The borders consisted of the walls of the left atrium excluding pulmonary veins and left atrial appendage. The biplane method of disks was used to calculate LA volume. LAVI was calculated by dividing LA volume by body surface area of subjects (Paul et al., 2010).

Peak transmitral flow $\mathrm{E}$ and $\mathrm{A}$ wave velocity, $\mathrm{E}$ wave deceleration time, were 
measured from the apical 4-chamber view. The LV ejection fraction (LVEF) was measured by Simpson's biplane method from the apical four- and two-chamber views. Pulsed wave Doppler was used to measure transmitral flow peak velocities in early (E) and late diastole (A), E/A ratio and E-wave deceleration time (DT) by placing the sample volume at the mitral leaflet tips in the apical four-chamber view. Tissue Doppler imaging was used to measure the peak velocity in early (E0) and late (A0) diastole with the sample volume placed at the lateral annulus. In this study, we used lateral mitral annulus velocity instead of the septal annulus velocity in view of the adverse effect of pulmonary hypertension on the interventricular septum. The E/E0 ratio was calculated as an estimate of $\mathrm{LV}$ diastolic pressure (Ommen et al., 2010). Additionally, lateral E0 $<10.0 \mathrm{~cm} / \mathrm{s}$ was used to define impaired LV myocardial relaxation (Nagueh et al., 2010).

Speckle tracking echocardiography STE images were acquired using a Philips ultrasound machine (Philips Medical Systems) with a broadband transducer (S5-1, 2-5 MHz). The images were coupled with an electrocardiographic record. Two dimensional speckle tracking strain (S) and strain rate (SR) analysis were performed, using special software (QLAB, version 8.1; Philips Medical Systems) with an LA zoomed view from the apical four chamber view and acquired at $>70$ fps. The LA endocardium was manually drawn in end systole; thereafter the endocardial borders were automatically tracked by the computer software throughout the cardiac cycle.

The LA myocardial width was adjusted by varying the thickness of the region of interest (ROI) applied to track the LA borders. The LA myocardium was divided into six segments (basal, middle and apical segments of the atrial septum and lateral wall, respectively) and LA apex segment. The software displayed peak longitudinal left atrial systolic strain (S) for each of the seven individual segments and the averaged global strain (Figure 1: a). The systolic (SRs), early diastolic (SRe) and late diastolic (SRa) longitudinal strain rates were determined as the averaged global LA strain rates from seven individual segments (Figure 1: b).
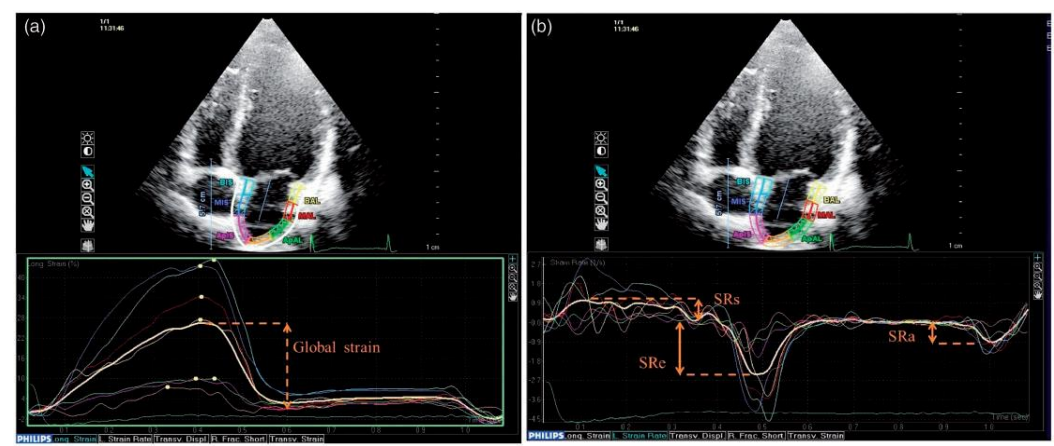

Figure (1): Measurement of left atrial strain (a) and strain rate (b). Abbreviations: (a) Global strain: global atrial longitudinal systolic strain. (b) SRs: global atrial longitudinal strain rate during ventricular systole; SRe: global atrial longitudinal strain rate during ventricular early diastole; SRa: global atrial longitudinal strain rate during ventricular late diastole. 
LA SRs was measured during LA passive stretching and left ventricular systole, which was taken as an index of LA reservoir function (Cianciulli et al., 2010). Similarly, SRe and SRa were measured during early and late diastole as indices of LA conduit and contractile function, respectively (Eshoo et al., 2011).

\section{Statistical analysis:}

Data were statistically described in terms of range, mean \pm standard deviation $( \pm$ SD), frequencies (number and percentages of cases) when appropriate. Differences in baseline characteristics between patients with and without the primary end point were assessed using Student t test, Mann-Whitney U test, or $\chi 2$ when appropriate. o compare categorical data, Pearson Chi square (X2) test was performed. Fisher's exact twotailed probability test was used instead when the expected frequency is less than 5. Correlation between various variables was done using Spearman rank correlation equation for non-normal variables. $\mathrm{P}$ values $<0.05$ were considered significant. All calculations were done using SPSS computer programs (Statistical Package for the Social Sciences; SPSS Inc., Chicago, IL, USA) version 23.

\section{RESULTS}

Sixty SLE patients aged 20 to 40 with normal LV systolic function (LVEF > $50 \%$ ). SLE patients were enrolled in this study. Healthy forty subjects were included in this study as controls. Subjects were divided into two groups: Group I; this group will include (60) individuals with SLE. Group II; this group will include (40) healthy individuals. Patients group were $54(90 \%)$ females and $6(10 \%)$ males, their mean ages was 32.23 \pm 5.26 years). The controls were $32(80 \%)$ females and $8(20 \%)$ males; their mean age was 31.82 \pm 5.06). There was no statistically significant difference between patients and control groups as regarding age, gender, BSA, SBP and DBP (Table 1).

Table (1): Demographic data of the studied groups

\begin{tabular}{|c|c|c|c|c|c|}
\hline \multicolumn{2}{|c|}{ Variables } & Groups & $\begin{array}{l}\text { Patient group } \\
(n=60)\end{array}$ & $\begin{array}{c}\text { Control group } \\
(n=40)\end{array}$ & $\mathbf{P}$ \\
\hline \multirow{4}{*}{ 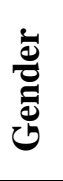 } & \multirow{2}{*}{ Male } & $\mathrm{n}$ & 6 & 8 & \multirow{4}{*}{$>0.05$} \\
\hline & & $\%$ & $10 \%$ & $20 \%$ & \\
\hline & \multirow{2}{*}{ Female } & $\mathrm{n}$ & 54 & 32 & \\
\hline & & $\%$ & $90 \%$ & $80 \%$ & \\
\hline \multicolumn{2}{|c|}{ Age (ys) } & Mean \pm SD & $32.23 \pm 5.26$ & $31.82 \pm 5.06$ & $>0.05$ \\
\hline \multicolumn{2}{|c|}{ BSA (m2) } & Mean \pm SD & $1.82 \pm 0.43$ & $1.73 \pm 0.09$ & $>0.05$ \\
\hline \multicolumn{2}{|c|}{ SBP $(\mathrm{mmHg})$} & Mean \pm SD & $123.65 \pm 16.74$ & $121.34 \pm 20.72$ & $>0.05$ \\
\hline \multicolumn{2}{|c|}{ DBP $(\mathrm{mmHg})$} & Mean \pm SD & $76.90 \pm 9.35$ & $78.02 \pm 4.72$ & $>0.05$ \\
\hline
\end{tabular}

$\mathrm{BSA}=$ Body surface area $(\mathrm{m} 2), \mathrm{SBP}=$ Systolic blood pressure $(\mathrm{mmHg}), \mathrm{DBP}=$ Diastolic blood pressure. 
LA dimension was not significantly different between both groups ( $p>0.05$ ). The LAVI was significantly larger in the SLE patients group than that in control group $(34.57 \pm 7.11$ vs. $27.63 \pm 9.25 \mathrm{ml} / \mathrm{m} 2$, $\mathrm{p}=0.031)$. LVEDD and LVESD and LVEF were similar in the SLE group and controls (Table 2).

Table (2): Comparison between the patients and the control groups regarding conventional echocardiographic features

\begin{tabular}{|l|c|c|c|}
\hline \multirow{2}{*}{ Groups } & $\begin{array}{c}\text { Patients group } \\
(\mathbf{n = 6 0})\end{array}$ & $\begin{array}{c}\text { Control group } \\
(\mathbf{n = 4 0})\end{array}$ & \multirow{2}{*}{$\boldsymbol{P}$-value } \\
\cline { 2 - 3 } & Mean \pm SD & Mean \pm SD & \\
\hline LA dimension $(\mathbf{m m})$ & $35.23 \pm 4.02$ & $33.80 \pm 4.63$ & $>0.05$ \\
\hline LAVI $(\mathbf{m L} / \mathbf{m}$ ) & $34.57 \pm 7.11$ & $27.63 \pm 9.25$ & $<\mathbf{0 . 0 0 1}$ \\
\hline LV end-diastolic dimension $(\mathbf{m m})$ & $48.54 \pm 4.54$ & $47.94 \pm 4.98$ & $>0.05$ \\
\hline LV end-systolic dimension $(\mathbf{m m})$ & $31.37 \pm 3.49$ & $31.22 \pm 3.84$ & $>0.05$ \\
\hline LV ejection fraction $(\%)$ & $65.41 \pm 3.97$ & $64.24 \pm 4.82$ & $>0.05$ \\
\hline
\end{tabular}

LA: left atrium; LV: left ventricle; LAVI: maximum left atrial volume indexed to BSA.

LV diastolic function parameters including peak $\mathrm{E}$ and $\mathrm{A}$ velocities, $\mathrm{E} / \mathrm{A}$ ratio, DT, and $\mathrm{E} / \mathrm{E} \backslash$ were not significantly different between patients and control groups $(p>0.05)$. Diastolic function was normal in 19(31.66\%), impaired relaxation $16(26.7 \%)$, pseudo normal $23(38.33 \%)$ and restrictive $2(3.34 \%)$ (Table 3).

Table (3): Comparison between the patients and the control groups regarding LV diastolic function

\begin{tabular}{|c|c|c|c|c|c|}
\hline \multicolumn{2}{|l|}{ Variables } & $\begin{array}{l}\text { Patients } \\
\text { group } \\
(n=60)\end{array}$ & $\begin{array}{c}\text { Control } \\
\text { group } \\
(\mathrm{n}=40)\end{array}$ & $\begin{array}{c}\text { Mann } \\
\text { Whitney } \\
\text { U test }\end{array}$ & $P$-value \\
\hline \multicolumn{2}{|l|}{$\begin{array}{l}\mathbf{E}(\mathbf{c m} / \mathbf{s}) \\
\text { mean } \pm \text { SD }\end{array}$} & $77.37 \pm 18.30$ & $82.70 \pm 3.90$ & - & $>0.05$ \\
\hline \multicolumn{2}{|c|}{$\begin{array}{l}\text { median } \\
\text { Min. - Max. }\end{array}$} & $\begin{array}{c}70.50 \\
(47-82.5) \\
\end{array}$ & $\begin{array}{c}69.10 \\
(47.1-79.7) \\
\end{array}$ & 0.971 & 0.331 \\
\hline \multicolumn{2}{|c|}{$\begin{array}{l}\text { E/A ratio } \\
\text { mean } \pm \text { SD }\end{array}$} & $1.35 \pm 0.31$ & $1.33 \pm 0.24$ & - & $>0.05$ \\
\hline \multicolumn{2}{|l|}{$\mathrm{E} \backslash(\mathrm{cm} / \mathrm{s})$} & $10.01 \pm 1.82$ & $11.72 \pm 2.42$ & - & $>0.05$ \\
\hline \multicolumn{2}{|c|}{$\begin{array}{l}\text { E/ E\ ratio } \\
\text { median } \\
\text { Min. - Max. }\end{array}$} & $\begin{array}{c}8.72 \\
(5.9-16.11) \\
\end{array}$ & $\begin{array}{c}7.83 \\
(5.56-16) \\
\end{array}$ & 1.555 & 0.120 \\
\hline \multicolumn{2}{|c|}{ Deceleration time (ms) } & $167.58 \pm 31.59$ & $159.10 \pm 30.83$ & - & $>0.05$ \\
\hline \multirow{4}{*}{$\begin{array}{c}\text { Diastolic } \\
\text { filling } \\
\text { pattern }\end{array}$} & Normal, n (\%) & $19(31.66 \%)$ & $37(92.5 \%)$ & & \multirow{4}{*}{0.001} \\
\hline & $\begin{array}{c}\text { Impaired relaxation, } \\
\mathrm{n}(\%)\end{array}$ & $16(26.7 \%)$ & $2(5 \%)$ & & \\
\hline & $\begin{array}{c}\text { Pseudo normal, } \mathrm{n} \\
(\%)\end{array}$ & $23(38.33 \%)$ & $1(2.5 \%)$ & & \\
\hline & Restrictive, n (\%) & $2(3.34 \%)$ & $0(0 \%)$ & & \\
\hline
\end{tabular}

E: mitral early diastolic inflow velocity; A: mitral late diastolic inflow velocity; É: early diastolic mitral annular tissue Doppler velocity; A: late diastolic mitral annular tissue Doppler velocity; LA: left atrium; LV: left ventricle. 
In this study, global strain, derived as median (range) of all segments, was 22.4(16-28.7) in SLE group vs. 28(16.334.2) in control group ( $\mathrm{p}=0.001)$. Septal, lateral and apical longitudinal strains were reduced in SLE patients with significantly differences between both groups. There was no significant difference in SRs between SLE and control groups 2(1.77$2.99)$ vs. $1.95(1.45-2.70), \mathrm{p}>0.0)$. LA SRe was decreased in the SLE group compared with that in control group -2.15(1.76-2.7) vs. -2.92(1.66-3.21), p<0.05). LA SRa was increased in SLE group compared with that in control group (-1.85(1.78-3.0) vs. -1.42(1.36-2.47), $\mathrm{p}<0.05)$ (Table 4).

Table (4): Comparison between the patients and the control groups regarding speckle tracking parameters

\begin{tabular}{|c|c|c|c|c|}
\hline \multirow[b]{2}{*}{ Variables } & $\begin{array}{c}\text { Patients group } \\
(n=60)\end{array}$ & $\begin{array}{c}\text { Control group } \\
(n=40)\end{array}$ & \multirow{2}{*}{$\begin{array}{c}\text { Mann } \\
\text { Whitney } \\
\text { U test }\end{array}$} & \multirow{2}{*}{$\begin{array}{c}P- \\
\text { value }\end{array}$} \\
\hline & $\begin{array}{c}\text { Median } \\
(\text { min-max })\end{array}$ & $\begin{array}{c}\text { Median } \\
(\text { min-max })\end{array}$ & & \\
\hline Global strain (\%) & $22.4(16-28.7)$ & $28(16.3-34.2)$ & 4.426 & 0.001 \\
\hline Basal septum strain (\%) & $25.3(17.6-31.2)$ & $30.6(18.2-37.4)$ & 2.931 & 0.003 \\
\hline Mid septum strain (\%) & $33.5(21.3-38.4)$ & $41.6(23.1-44.8)$ & 5.231 & $<0.001$ \\
\hline Apical septum strain (\%) & $14.7(9.32-18.3)$ & $18.63(8.76-22.5)$ & 2.431 & $\mathbf{0 . 0 2 7}$ \\
\hline Basal lateral strain (\%) & $23.6(11.32-26.2)$ & $32.8(14.22-35.70)$ & 4.542 & $<0.001$ \\
\hline Mid lateral strain (\%) & $29.2(16.45-33.61)$ & $37.8(17.32-40.11)$ & 4.335 & $<0.001$ \\
\hline Apical lateral strain (\%) & $7.52(5.34-11.30)$ & $15.14(6.22-18.61)$ & 5.147 & $<0.001$ \\
\hline Apex strain (\%) & $5.7(3.64-6.33)$ & $9.42(3.73-11.3)$ & 3.892 & $<0.001$ \\
\hline SRs & $2(1.77-2.99)$ & $1.95(1.45-2.70)$ & 0.885 & 0.37 \\
\hline SRe & $-2.15(1.76-2.7)$ & $-2.92(1.66-3.21)$ & 2.305 & 0.025 \\
\hline SRa & $-1.85(1.78-3.0)$ & $-1.42(1.36-2.47)$ & 2.152 & 0.032 \\
\hline
\end{tabular}

SRs: systolic strain rate; SRe: early diastolic strain rate; SRa: late diastolic strain rate.

Left ventricular diastolic dysfunction (LVDD) SLE groups was compared as normal or abnormal diastolic function (including normal, impaired relaxation and pseudo-normal and restrictive groups) using Mann Whitney U test. There were significant differences in LAVI, SRs, SRe and SRa among normal and abnormal diastolic function in patients of the SLE group $(\mathrm{p}<0.05)$ (Table 5).

Table (5): Differences in left atrial parameters of SLE patients based on diastolic function

\begin{tabular}{|c|c|c|c|c|}
\hline & \multicolumn{2}{|c|}{$\begin{array}{c}\text { Diastolic function } \\
\text { in patients group }(n=60)\end{array}$} & \multirow{3}{*}{$\begin{array}{l}\text { Mann Whitney } \\
\text { U test }\end{array}$} & \multirow{3}{*}{$P$-value } \\
\hline & Normal & Abnormal & & \\
\hline & $\begin{array}{c}\text { Median } \\
(\text { min-max) }\end{array}$ & $\begin{array}{c}\text { Median } \\
(\text { min-max })\end{array}$ & & \\
\hline $\mathrm{LAVI}(\mathrm{ml} / \mathrm{m} 2)$ & $29.6(26.0-33.7)$ & $34.1(27.3-37.2)$ & 2.240 & 0.019 \\
\hline SRs & $2.10(1.85-2.99)$ & $1.73(1.77-2.35)$ & 3.271 & 0.01 \\
\hline SRe & $-2.41(1.88-2.7)$ & $-1.96(1.76-2.34)$ & 4.863 & $0<0.001$ \\
\hline SRa & $-1.80(1.78-2.48)$ & $-2.25(2.06-3.0)$ & 4.529 & $0<0.001$ \\
\hline
\end{tabular}

SRs: systolic strain rate; SRe: early diastolic strain rate; SRa: late diastolic strain rate. 
In this table, the correlation between LVDD and left atrial SRs, SRe and, SRa showed that there were significant positive correlations with SRs $(r=0.329$, $\mathrm{p}=0.010), \operatorname{SRe}(\mathrm{r}=0.274, \mathrm{p}=0.034)$ but not significant with $\mathrm{SRa}(\mathrm{r}=0.197, \mathrm{p}=0.131)$ (Table 6).

Table (6): Correlation between LVDD and left atrial SRs SRe and SRa

\begin{tabular}{|l|c|c|}
\hline LVDD Correlation & r & $\boldsymbol{P}$-value \\
\hline SRs & 0.329 & $\mathbf{0 . 0 1 0}$ \\
\hline SRe & 0.274 & $\mathbf{0 . 0 3 4}$ \\
\hline SRa & -0.197 & $>0.05$ \\
\hline
\end{tabular}

\section{DISCUSSION}

Patients group were $90 \%$ females and $10 \%$ males, their mean ages was $32.23 \pm 5.26$ years. The controls were $80 \%$ females and $20 \%$ males. Their mean age was 31.82 \pm 5.06. Mok (2011) reported that Systemic lupus erythematosus (SLE) is a chronic autoimmune disease that mostly affects young women.

In this study, there was no statistically significant difference between patients and control groups as regarding age, gender and BSA. Our results showed no significant differences between both groups. This can help in exclusion of effect of age and BSA on left atrial function. Some studies have shown a small yet significant relationship between age and LA strain and strain rate (Gan et al., 2018). Morris et al. (2015) showed the difference in reservoir function persisted, but there was no difference in contractile function. A study demonstrated that with increasing body surface area, there were significant decreases in reservoir strain and contractile strain (Pathan et al., 2017).

In this study, LA dimension was not significantly different between both groups. LVEDD and LVESD and LVEF were similar in the SLE group and controls. The LAVI was significantly larger in the SLE patients group than that in control group. Our findings were in line with Dai et al. (2016) who found that the LAVI was significantly larger in the SLE group than that in control group. This can be explained by elevations of left intraventricular pressure during diastole, increasing the load on the left atrium (LA) and inducing the LA remodeling (Hsiao et al., 2012). Also, in accordance with Kucuk et al. (2018), conventional echocardiography parameters were not statistically different between groups.

These results suggested that LA structural change was also influenced by LVDD. Due to the thinness of the LA wall, it is easily influenced by pressure and volume load. When left ventricular diastolic function is impaired, enddiastolic pressure increases, and consequently, LA pressure load will increase, leading to LA enlargement (Shang et al., 2012).

STE analysis has been demonstrated to be a feasible method to assess global and phasic LA longitudinal strains and strain rates in healthy subjects and in patients with LA dysfunction (Kurt et al., 2014). SRs has been used as an index of LA reservoir function (Cianciulli et al., 2010), 
while SRe and SRa are accepted as indices of LA conduit and LA contractile function, respectively (Eshoo et al., 2011).

In this study, global strain was reduced in SLE group vs. control group. Septal, lateral and apical longitudinal strains reduced in SLE patients with significant differences between both groups. LA SRe was decreased in the SLE group compared with that in control group. LA SRa was increased in SLE group compared with that in control group. When the left ventricular diastolic function was normal in the SLE group, there were no significant differences in SRs, SRe and SRa between the normal diastolic function group in SLE and the control group.

The reduction in LA SRe and increase in positive $\mathrm{SRa}$ indicated that the changes in LA function of SLE patients were likely characterized by impaired conduit and enhanced booster pump functions. The source of SLE-altered atrial myocardium deformation dynamics via left ventricular diastolic dysfunction and stiffness of the atrial myocardium fibres may be the chronic inflammatory state and immune complex deposition (GuettrotImbert et al., 2011).

According to the Dai et al. (2016) results of STE showed positive SRe decrease (conduit function) and positive SRa increase (pump function). In pseudo normal and restrictive group, left ventricular relaxation and compliance all fell, early and late diastolic pressure increased, and LA pump function was beyond the compensatory limit, and declined in late diastole. Positive SRe and SRa were both decreased. The above results demonstrate that left ventricular diastolic dysfunction plays a progressive role in the deterioration of LA function. Similarly our results demonstrated that LA global S, SRs and positive SRe were reduced in SLE patients.

Left ventricular diastolic dysfunction (LVDD) in control and SLE groups was compared as normal or abnormal diastolic function (including normal, impaired relaxation and pseudo-normal and restrictive groups). This means that LA deformation dynamics were impaired in SLE patients compared with age-matched and gender-matched controls, the magnitude of LA impairment was increased with the severity of LVDD. In agreement with Dai et al. (2016), they found that left atrial peak systolic strains were reduced in all seven segments of the SLE group and Global strain was decreased in SLE group vs control healthy group.

Acar et al. (2015) found significantly decreased values of the left atrial peak systolic strain by using 2-dimensional speckle tracking echocardiography and larger volumes of the LA than the control group. Kurt et al. (2014) were demonstrated that diastolic heart failure is associated with larger LA volumes and decreased LA peak systolic strain. The major problem caused an increase in LA volume and decrease in LA peak systolic strain in patients with SLE may be the reduced diastolic reserve due to the abnormal myocardial relaxation. During diastole, the greater LV filling pressure reflects the greater pressure gradient vs. the left atrium, which in turn is a consequence of atrial enlargement (Hsiao et al., 2012).

Also, Perez-Topete et al. (2019) reported that SLE patients had lower 
myocardial deformation of the LA, which is expressed as a lower diastolic function correlating with early subclinical myocardial damage. Several studies have described LV diastolic dysfunction in SLE patients, including that it is more pronounced in patients with active disease (Teixeira et al., 2010 and Chen et al., 2016).

In this study, the correlation between LVDD and left atrial SRs and SRe were significant correlations. In consistent with Morris et al. (2015) who reported that LA strain has been shown to correlate with the degree of diastolic dysfunction. By comparing 329 normal adults to 377 adults with diastolic dysfunction. They demonstrated that LA function derived both volumetrically and by 2D STE strain, was inversely related to $\mathrm{LV}$ filling pressure (mitral $\mathrm{E} / \mathrm{E}^{\prime}$ ratio) and to the degree of LV diastolic dysfunction.

Also, Guan et al. demonstrated significant changes in LA strain rate parameters between different grades of LV diastolic dysfunction in subjects with preserved LVEF, despite no significant difference in LA volume between the groups (Guan et al., 2010). Compared with patients with normal diastolic function, patients with mild diastolic dysfunction had significantly reduced $\mathrm{E}$ sr and $\mathrm{S}$ sr but increased A sr. Another study Cameli et al. (2016) demonstrated that LA systolic strain (measured using STE) inversely correlated with $\mathrm{LV}$ end diastolic pressure. Similar to the E/E' ratio, LA systolic strain correlates with LV end diastolic pressure in patients with preserved or mildly reduced LVEF, whereas in patients with moderate or severe reduction of $\mathrm{EF}, \mathrm{E} / \mathrm{E}^{\prime}$ correlated poorly and LA systolic strain provided a better estimation of LV filling pressures.

\section{CONCLUSION}

This study has reported that LA function was impaired in SLE patients. The changes in the LA deformation, dynamic function and volume of SLE patients were characterized by impaired conduit, decreased storage function and enhanced auxiliary pump activities. Left atrial remodeling and impaired LA mechanical functions can be detected accurately with speckle tracking echocardiography. Assessment of LA strain represents a simple, accurate and reproducible technique to evaluate LA function. LA strain and strain rate parameters are more sensitive than conventional parameters of atrial function. Strain parameters demonstrate alterations prior to alterations in LA volumes with new data regarding its prognostic relevance emerging rapidly.

\section{REFERENCES}

1. Acar RD, Bulut M, Acar Ş, Izci S, Fidan S, Yesin $M$ and Efe SC. (2015): Evaluation of the $\mathrm{P}$ wave axis in Patients with Systemic Lupus Erythematosus. J Cardiovasc Thorac Res., 7(4):154-157.

2. Cameli M, Sparla $S$ and Losito $M$. (2016): Correlation of Left Atrial Strain and Doppler Measurements with Invasive Measurement of Left Ventricular EndDiastolic Pressure in Patients Stratified for Different Values of Ejection Fraction. Echocardiography, 33:398-405.

3. Chen J, Tang Y, Zhu $M$ and Xu A. (2016): Heart involvement in systemic lupus erythematosus: a systemic review and meta-analysis. Clin Rheumatol., 35(10):2437-2448. 
4. Cianciulli TF, Saccheri MC, Lax JA, Bermann AM and Ferreiro DE. (2010): Two-dimensional speckle tracking echocardiography for the assessment of atrial function. World J Cardiol., 2: 163170.

5. Dai M, Li KL and Qian DJ. (2016): Evaluation of left atrial function by speckle tracking echocardiography in patients with systemic lupus erythematosus. Lupus, 25(5):496-504.

6. Eshoo S, Semsarian C, Ross DL, Marwick TH and Thomas L. (2011): Comparison of left atrial phasic function in hypertrophic cardiomyopathy versus systemic hypertension using strain rate imaging. Am J Cardiol., 107: 290-296.

7. Gan GC, Ferkh A, Boyd A and Thomas L. (2018): Left atrial function: evaluation by strain analysis. Cardiovasc Diagn Ther., 8:29-46.

8. Guan Z, Zhang D and Huang R. (2010): Association of left atrial myocardial function with left ventricular diastolic dysfunction in subjects with preserved systolic function: a strain rate imaging study. Clin Cardiol., 33:643-9.

9. Guettrot-Imbert G, Cohen $L$ and Fermont L. (2011): A new presentation of neonatal lupus: 5 cases of isolated mild endocardial fibroelastosis associated with maternal Anti-SSA/Ro and Anti-SSB/La antibodies. J Rheumatol., 38: 378-386.

10. Hesselvig JH, Ahlehoff O, Dreyer L, Gislason $G$ and Kofoed K. (2017): Cutaneous lupus erythematosus and systemic lupus erythematosus are associated with clinically significant cardiovascular risk: a Danish nationwide cohort study. Lupus, 26(1):48-53.

11. Hsiao SH, Lin KL and Chiou KR. (2012): Comparison of left atrial volume parameters in detecting left ventricular diastolic dysfunction versus tissue
Doppler recordings. Am J Cardiol., 109: 748-755.

12. Kucuk M, Korucuk N, Tosun V, Cavusoglu $M$ and Basarici İ. (2018): Assessment of left atrial function using speckle tracking echocardiography in ankylosing spondylitis: a case-control study. Int J Cardiovasc Imaging, 34(12):1863-1868.

13. Kurt M, Tanbog ${ }^{-}$a IH and Bu" yu“ kkaya E. (2014): Relation of presence and severity of metabolic syndrome with left atrial mechanics in patients without overt diabetes: a deformation imaging study. Anadolu Kardiyol Derg., 14: 128133.

14. Liu Y, Wang $K$ and Su D. (2014): Noninvasive assessment of left atrial phasic function in patients with hypertension and diabetes using twodimensional speckle tracking and volumetric parameters. Echocardiography, 31:727-35.

15. Mok CC. (2011): Epidemiology and survival of systemic lupus erythematosus in Hong Kong Chinese. Lupus, 20(7):767771.

16. Morris DA, Takeuchi $M$ and Krisper M. (2015): Normal values and clinical relevance of left atrial myocardial function analysed by speckle-tracking echocardiography: multicentre study. Eur Heart J Cardiovasc Imaging, 16:364-72.

17. Nagueh SF, Appleton CP and Gillebert TC. (2010): Recommendations for the evaluation of left ventricular diastolic function by echocardiography. J Am Soc Echocardiogr., 22: 107-133.

18. Obokata M, Negishi $K$ and Kurosawa K. (2014): Left atrial strain provides incremental value for embolism risk stratification over CHA-DS--VASc score and indicates prognostic impact in 
patients with atrial fibrillation. J Am Soc Echocardiogr., 27:709-716.e4.

19. Ommen SR, Nishimura RA and Appleton CP. (2010): Clinical utility of doppler echocardiography and tissue doppler imaging in the estimation of left ventricular filling pressures. Circulation, 102: 1788-1794.

20. Pathan F, D'Elia $\mathbf{N}$ and Nolan MT. (2017): Normal Ranges of Left Atrial Strain by Speckle-Tracking Echocardiography: A Systematic Review and Meta-Analysis. J Am Soc Echocardiogr., 30:59-70.e8.

21. Paul B. (2010): Left atrial volume - A New Index in Echocardiography. JAPI., 57:463-467.

22. Perez-Topete S, Miranda-Aquino T, Hernandez-del Rio J, Gutierrez-Ure. S, Martinez-Bonilla $\mathbf{G}$ and Gonzalez-Diaz V. (2019): Evaluation of left atrial myocardial deformation as marker of subclinical damage in patient with systemic lupus erythematosus. Ann Rheum Dis, 19: 45-52.
23. Shang Q, Yip GW and Tam LS. (2012): SLICC/ACR damage index independently associated with left ventricular diastolic dysfunction in patients with systemic lupus erythematosus. Lupus, 21: 10571062.

24. Teixeira AC, Bonfá E, Herskowictz N, Barbato AJ and Borba EF. (2010): Early detection of global and regional left ventricular diastolic dysfunction in systemic lupus erythematosus: the role of the echocardiography. Rev Bras Reumatol., 50(1):16-30.

25. Vianna-Pinton R, Moreno CA, Baxter CM, Lee KS, Tsang TS and Appleton CP. (2010): Two-dimensional speckletracking echocardiography of the left atrium: feasibility and regional contraction and relaxation differences in normal subjects. J Am Soc Echocardiogr., 22:299-305.

26. Vieira MJ, Teixeira R, Gonçalves $L$ and Gersh BJ. (2014): Left atrial mechanics: echocardiographic assessment and clinical implications. J Am Soc Echocardiogr., 27:463-78. 


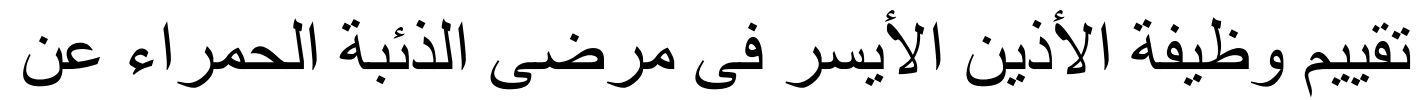
طريق التتبع النقطى بالموجات فوق الصنية فئية ثنائية الأبعاد على القلب

عبد القوي عبد الحليم أحمد، ممدوح حلمي الطحان، عبدالحميد عبدالحارث غزاليث،

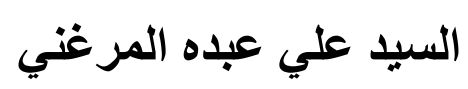

قسمي أمراض القلب، الطب الطبيعي والروماتيزم و التأهيل*، كلية الطب، جامعة الأزهر

E-mail: $\underline{\text { abdelkawy.abdelhalem@gmail.com }}$

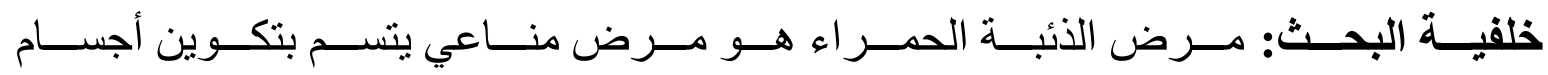

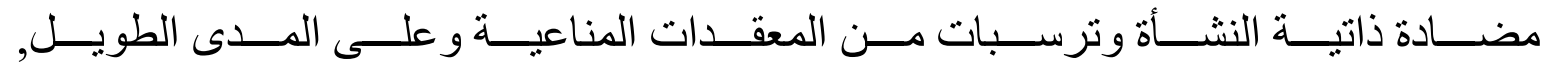

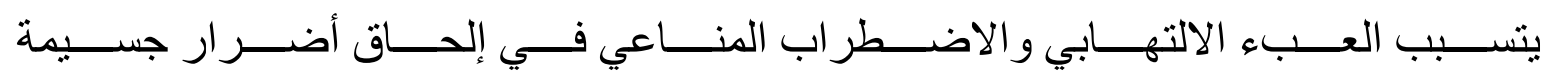

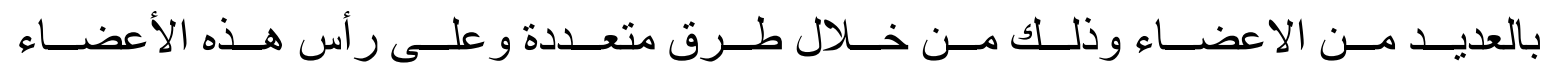

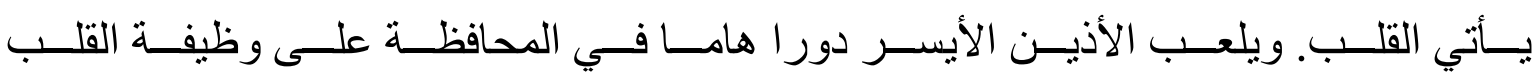

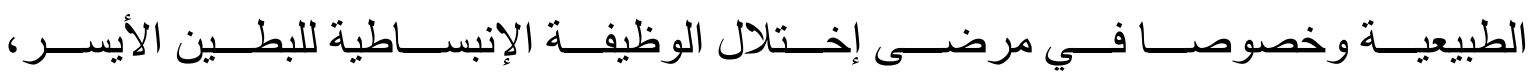

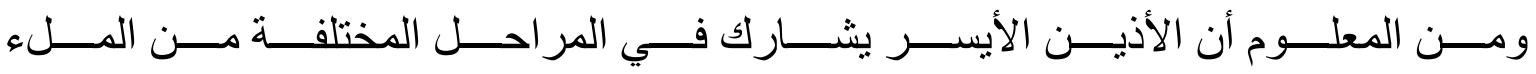

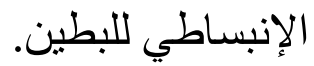

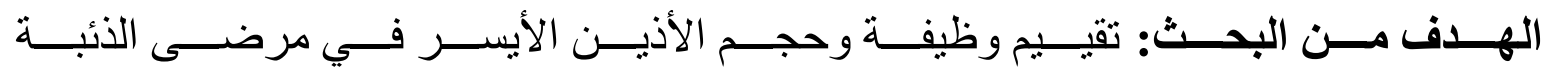

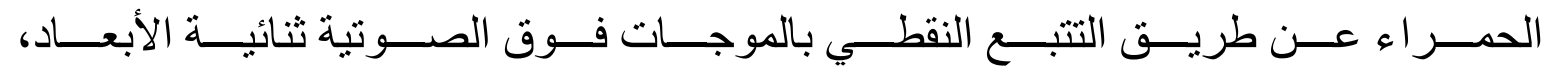

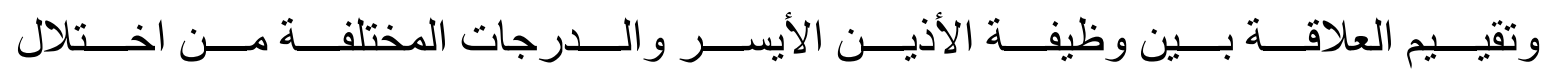
الوظيفة الإنبساطية للبطين الأيسر في مرضى الذئبة الحمراء.

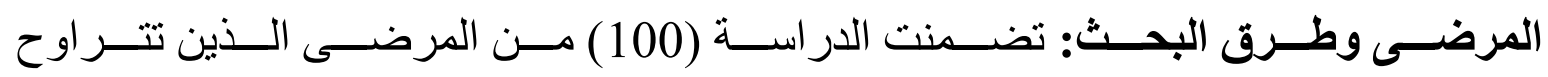

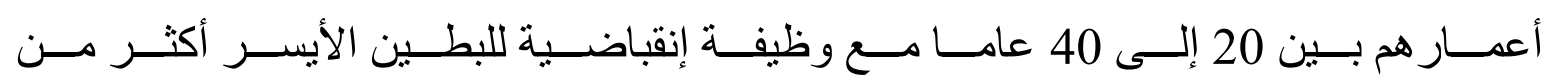

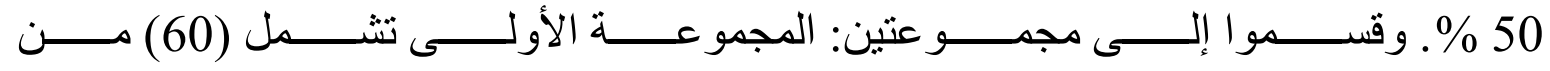

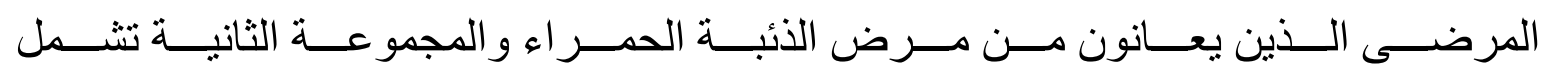

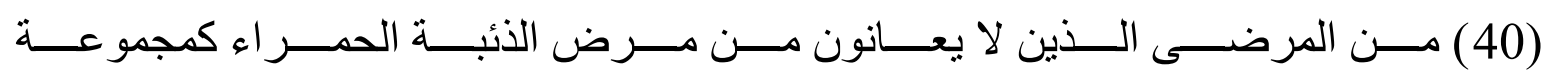
ضابطة تتناسب من حيث السن و النوع مع المرضى قيد الدراسة. 


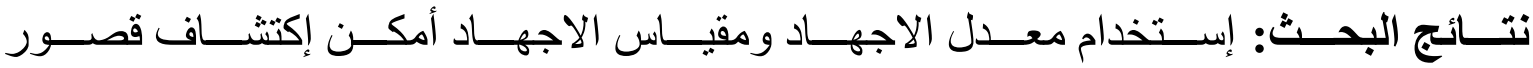

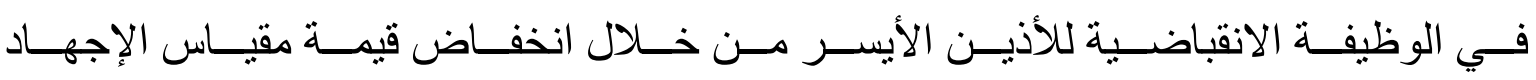

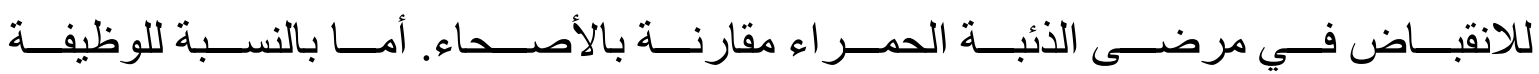

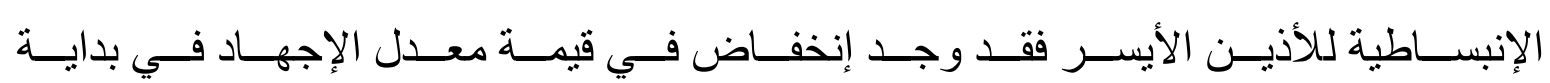

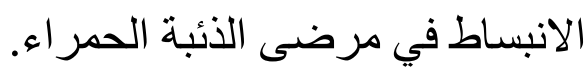

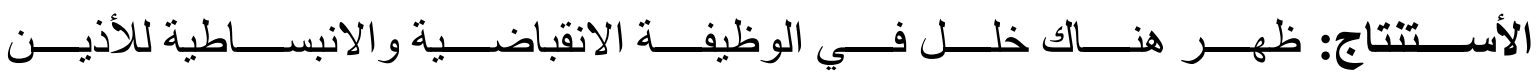

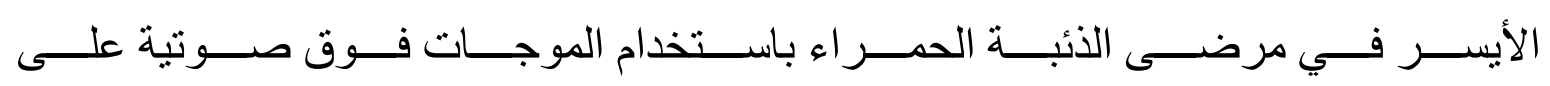

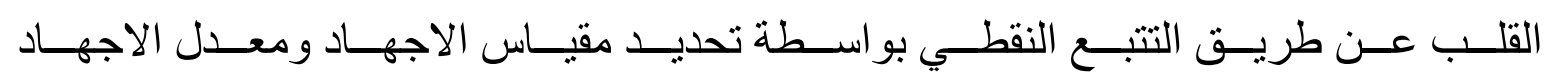

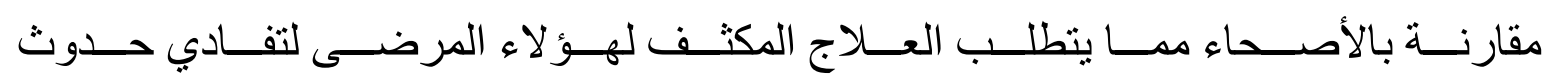
ضعف بعضلة القلب.

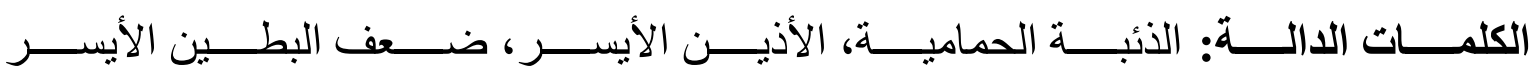
الانبساطي، تخطيط صدى القلب. 